Cite this: J. Mater. Chem. C, 2014, 2, 701

Received 11th July 2013

Accepted 11th November 2013

DOI: $10.1039 / \mathrm{c} 3 \mathrm{tc} 31333 f$

www.rsc.org/MaterialsC

\section{Homopolymers as nanocarriers for the loading of block copolymer micelles with metal salts: a facile way to large-scale ordered arrays of transition- metal nanoparticles}

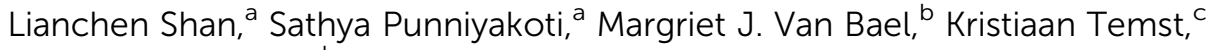
Marlies K. Van Bael, ${ }^{d}$ Xiaoxing Ke, ${ }^{e}$ Sara Bals, ${ }^{e}$ Gustaaf Van Tendeloo, ${ }^{e}$

Marc D'Olieslaeger, ${ }^{\text {af }}$ Patrick Wagner, ${ }^{\text {af }}$ Ken Haenen ${ }^{\text {af }}$ and Hans-Gerd Boyen*af

A new and facile approach is presented for generating quasi-regular patterns of transition metal-based nanoparticles on flat substrates exploiting polystyrene-block-poly2vinyl pyridine (PS- $b$-P2VP) micelles as intermediate templates. Direct loading of such micellar nanoreactors by polar transition metal salts in solution usually results in nanoparticle ensembles exhibiting only short range order accompanied by broad distributions of particle size and inter-particle distance. Here, we demonstrate that the use of P2VP homopolymers of appropriate length as molecular carriers to transport precursor salts into the micellar cores can significantly increase the degree of lateral order within the final nanoparticle arrays combined with a decrease in spreading in particle size. Thus, a significantly extended range of materials is now available which can be exploited to study fundamental properties at the transition from clusters to solids by means of well-organized, well-separated, size-selected metal and metal oxide nanostructures.
\end{abstract}

\section{Introduction}

Well-separated (i.e. non-interacting) inorganic nanostructures on surfaces continue to attract intense research interest since they allow the study of many of the fascinating properties of nanomaterials that can be distinctly different from their bulk counterparts. Among them, size-selected nanoparticles have already demonstrated their potential to address fundamental questions regarding, e.g., catalysis, ${ }^{1-3}$ chemical reactivity, ${ }^{4,5}$ magnetism, ${ }^{6}$ plasmonics, ${ }^{7,8}$ nanotube $^{9}$ and nanowire ${ }^{10,11}$ growth, the adhesion of cells,,$^{12,13}$ the alignment of DNA, ${ }^{14}$ and the exploitation as waveguides ${ }^{15}$ rendering themselves highly attractive for a large variety of applications. To effectively elaborate such issues and enable related applications, one of the key ingredients required is the ability to integrate a large number of size-selected nanoparticles into ordered arrays on a

\footnotetext{
${ }^{a}$ Institute for Materials Research (IMO), Hasselt University, Wetenschapspark 1, 3590 Diepenbeek, Belgium.E-mail: hansgerd.boyen@uhasselt.be

${ }^{b}$ Laboratory of Solid State Physics and Magnetism, KU Leuven, Celestijnenlaan 200D, 3001 Leuven, Belgium

'Instituut voor Kern- en Stralingsfysica, KU Leuven, Celestijnenlaan 200D, bus 2418, 3001 Leuven, Belgium

${ }^{d}$ Inorganic and Physical Chemistry, Institute for Materials Research, Hasselt University, Agoralaan Building D, 3590 Diepenbeek, Belgium

${ }^{e}$ Electron Microscopy for Materials Research (EMAT), University of Antwerp, Groenenborgerlaan 171, 2020 Antwerpen, Belgium

IImec vzw Division IMOMEC, Wetenschapspark 1, 3590 Diepenbeek, Belgium
}

technologically relevant substrate, where the particle size and inter-particle spacing can be tuned accordingly. To this end, exploiting the self-assembly of block copolymers (BCPs) provides bottom-up synthetic routes that are cost-effective in delivering nanostructures with characteristic dimensions below $20 \mathrm{~nm} .{ }^{16-22}$ The BCP-derived micellar technique ${ }^{19,23}$ makes use of precursor salt-loaded BCP micelles formed in a selective solvent as intermediate building blocks. This way, periodic ensembles of nanostructures can be achieved by transferring micellar monolayers to substrate surfaces. ${ }^{19,23,24}$ Here, convenient control over particle size and interparticle distance can be attained via the amount of precursor salt added to the micellar core and the length of the polymer chains forming the micellar nanoreactors. ${ }^{19,23}$ Yet, owing to the complex nature of the interaction between the precursor salt and the micellar nanoreactors in the solution, the preparation of ordered arrays of size-selected transition metal-based nanoparticles via this approach is still not well established, despite their technological relevance.

Here, we report a facile new approach which pushes the micellar method towards a wider spectrum of material systems including transition metals and their oxides. Whereas the conventional micellar technique involves directly adding an appropriate precursor salt to a solution containing pre-formed micellar assemblies, our new method makes use of pre-loaded homopolymers as molecular carriers for the homogeneous loading of micellar nanoreactors with the metal precursor. This 
leads to the deposition of size-selected inorganic nanoparticles into arrays with significantly increased medium-range order, even for transition metals. To demonstrate the new approach, two distinctly different transition-metal systems have been prepared (iron and niobium), motivated by their fundamental importance in magnetism and superconductivity, as well as the increasing potential of their oxides in applications related to photocatalysis, ${ }^{25-29}$ photovoltaics, ${ }^{30}$ biosensing, ${ }^{31}$ environmental remediation, ${ }^{32}$ and lithium ion batteries. ${ }^{33}$

\section{Experimental}

All polymers were purchased from Polymer Source Inc. (Canada). To prepare a typical solution, $25 \mathrm{mg}$ polystyrene-blockpoly2vinyl pyridine (PS- $b$-P2VP) was dissolved in $5 \mathrm{ml}$ toluene and stirred for 3 days at room temperature in order to allow the formation of reverse micelles. Simultaneously, $10 \mathrm{mg}$ homopolymer poly2vinylpyridine (hP2VP) was mixed with metal precursors in $1 \mathrm{ml}$ toluene and stirred for 3 days as well, to load the homopolymers by complexation. The precise amount of salt (which in fact controls the size of the final nanoparticles) was adjusted to yield a loading ratio (LR) of the hP2VP strands (ratio between the number of metal ions and pyridine units) of $\mathrm{LR}=0.3$ for $\mathrm{FeCl}_{3}$ and $\mathrm{LR}=0.18$ for $\mathrm{NbCl}_{5}$. Both values represent the maximum metal salt concentration which still allows deposition of micellar arrays with excellent hexagonal order. It is worth mentioning that both numbers are well below the loading ratio of 0.5 observed for the direct loading of micelles with metal salts. ${ }^{19,23}$

This resulting precursor salt-h2VP mixture was then added to the $5 \mathrm{ml}$ micellar solution and stirred for another week. In contrast, following the conventional approach, a metal salt was directly added to pre-formed micellar solutions and stirred for one more week afterwards. Si wafers were then dip-coated in the different solutions after filtration to produce micellar monolayers, ${ }^{19,23,24,34}$ which were subsequently exposed to radiofrequency (RF) oxygen plasma $\left(30 \mathrm{~W}, 0.02 \mathrm{mbar}, 250{ }^{\circ} \mathrm{C}\right)$ for $30 \mathrm{~min}$. This allowed removal of all organic parts of the micellar structures as proven by in situ chemical analysis using X-ray Photoelectron Spectroscopy (XPS). AFM measurements were carried out in air using a Digital Instrument Nanoscope IIIa SPM operated in tapping mode. Particle analysis including the calculation of the auto-covariance functions was performed using Nanoscope software version 5 .

\section{Results and discussion}

The challenge one has to face when using the conventional micellar technique for the deposition of transition metal-based nanoparticle ensembles is illustrated in Fig. 1a and b. Experimentally, a representative amphiphilic BCP system commonly used in this technique, PS- $b$-P2VP, with block lengths PS(32 500)- $b$-P2VP(7800) (the numbers in brackets indicate the molecular weight in $\mathrm{g} \mathrm{mol}^{-1}$ ) was dissolved in toluene to form reverse spherical micelles with PS forming the shell and P2VP forming the core. Subsequently, the micelles were loaded with metal salt precursors $\left(\mathrm{FeCl}_{3}\right.$ and $\left.\mathrm{NbCl}_{5}\right)$. Driven by the

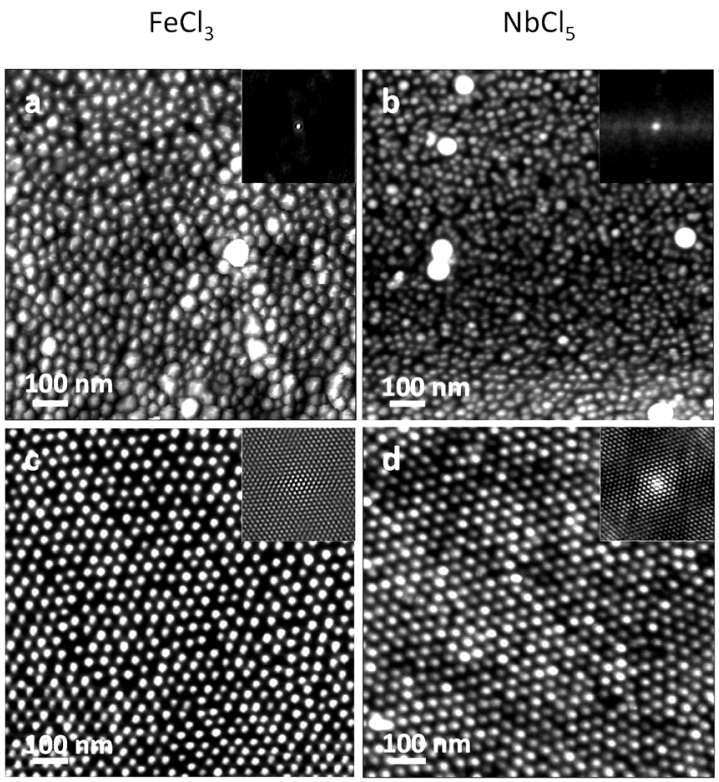

Fig. 1 AFM images demonstrating the poor lateral order of micellar monolayers on Si substrates loaded directly into solution with $\mathrm{FeCl}_{3}$ (a) and $\mathrm{NbCl}_{5}$ (b) metal precursors ('conventional' technique). Using our new approach, a significantly improved lateral order can be achieved for both $\mathrm{FeCl}_{3}$ (c) as well as $\mathrm{NbCl}_{5}$ (d) loaded micelles. The insets represent the auto-covariance functions of the corresponding AFM images.

incompatible polarity between toluene (nonpolar) and the metal salt (polar), the precursor ions diffuse into the micellar cores and interact through coordination bonds with several pyridine sites. Such polydentate interactions, including intraand inter-molecular cross-linking of P2VP blocks, ${ }^{35-37}$ result in strongly perturbed molecular conformations ${ }^{37}$ and thus might influence the morphology of the pristine (unloaded) micelles. Consequently, after their deposition onto a substrate, a large distribution in size and shape of micellar aggregates can be anticipated leading to irregular patterns lacking any degree of order. Such a behavior can indeed be observed in the AFM images presented in Fig. 1a and $\mathrm{b}$ for the two different precursor salts, with the corresponding auto-covariance functions lacking even short-range order (see insets).

To overcome this unsatisfactory situation, a new procedure has been developed as outlined in Scheme 1 . Firstly, PS- $b$-P2VP is dissolved in toluene and stirred for 3 days to allow the formation of reverse micelles (a). Simultaneously, hP2VP is mixed with an appropriate amount of metal precursor and toluene in a separate vial, also stirred for 3 days to allow complex formation between the metal salt ions and the pyridine units (b). Subsequently, the metal salt-loaded hP2VP solution is added to the BCP micellar solution and stirred for another week (c). Finally, a monolayer of homopolymer- and, thus, metal saltloaded micelles is deposited onto a Si substrate by dip-coating yielding ordered arrays of inorganic nanoparticles after a subsequent plasma treatment (d).

In order to allow a direct comparison between our new approach and the 'conventional' micellar technique, the same type of micellar solutions as presented in Fig. 1a and b (except 
(a)

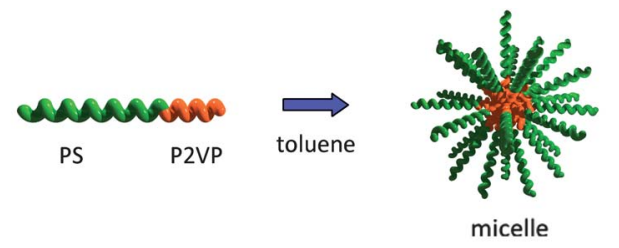

(b)

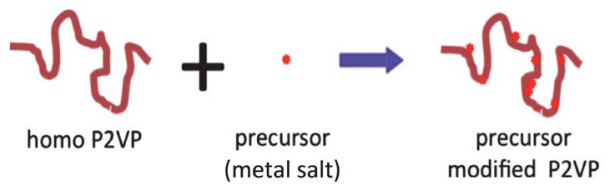

(c)

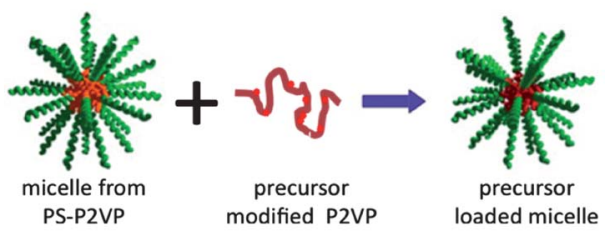

(d)

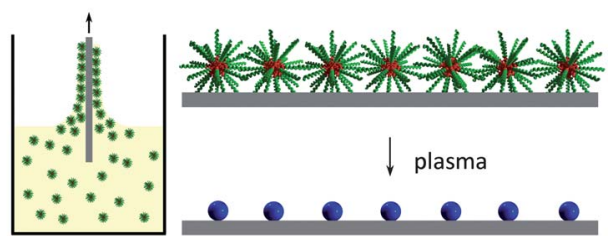

Scheme 1 Illustration of the basic steps of the new method: first, reverse micelles are formed from PS- $b$-P2VP diblock copolymers after dissolution in toluene (a); simultaneously, hP2VP strands are hybridized in a separate vial with an appropriate metal-salt precursor (b); subsequently, the micellar solution is mixed with the precursor-loaded hP2VP strands, which serve as nanocarriers to transfer the metal salt into the micellar cores (c); finally, the loaded micelles are transferred onto a substrate by dip-coating, resulting in ordered arrays of inorganic nanoparticles after plasma treatment (d).

their loading with metal salts) were prepared. Simultaneously, hP2VP(5000) was dissolved in toluene and loaded with $\mathrm{FeCl}_{3}$ $\left(\mathrm{NbCl}_{5}\right)$ salt according to the procedure depicted in Scheme $1 \mathrm{~b}$. Owing to its incompatibility with toluene, the homopolymer hP2VP(5000), being chemically identical to the polymer block forming the micellar core, is expected to easily diffuse into the micellar nanoreactors in order to reduce the total interfacial energy of the system. ${ }^{38}$ Once loaded with the $\mathrm{FeCl}_{3}\left(\mathrm{NbCl}_{5}\right)$ precursor salt, an even increased driving force for this diffusion is expected because of the polar character of the metal salt. As now the metal ion precursors are already coordinated beforehand by the homopolymer hP2VP, they are not expected to form additional coordination bonds with the P2VP blocks forming the micellar cores. This should help to avoid the morphological distortion observed in the case of direct loading. The resulting micellar monolayers (deposited onto Si substrates) derived via the new approach for both types of metal salts are presented in Fig. 1c and d. Clearly, a significantly improved array quality can be recognized exhibiting even hexagonal medium-range order as evidenced by the corresponding auto-covariance functions (see insets).

As the spatial distribution of homopolymer molecules within equivalent blocks in microphase-separated polymer blends strongly depends on their molecular weight, ${ }^{39-43}$ the impact of the chain length of the hP2VP precursor carriers on the lateral order of the resulting micellar monolayers has been studied in more detail. For this purpose, and to demonstrate the applicability of the new approach for larger micellar structures based on longer diblock-copolymer chains as well, a different BCP system of PS(185 000)-b-P2VP(90 000) is exploited. As homopolymers with molecular weights exceeding that of the equivalent block cause markedly decreased solubility within the microdomains and even lead to macro-phase separation, ${ }^{\mathbf{4 0 , 4 4}}$ two different degrees of polymerization were chosen for the hP2VP molecules, one with similar chain length hP2VP(97 000) and one with significantly shorter length hP2VP(1200). The resulting micellar arrays are presented in Fig. 2. Here, utilizing short hP2VP molecules as carriers for the homogeneous loading of micellar nanoreactors with transition metal-based salts is found to completely fail (Fig. 2a and b) as similarly observed in the case of direct loading (Fig. 1a and b). However, if a chain length is chosen for the homopolymer that matches the block length of the P2VP block forming the micellar core, excellent hexagonal medium-range order is obtained for both precursor salts (Fig. 2c and d). In order to demonstrate the significant improvement in array quality achievable using our new technique, an AFM image has been added (Fig. 2e) presenting the results shown in Fig. $2 \mathrm{c}$ at a larger scale $\left(10 \times 10 \mu \mathrm{m}^{2}\right)$. Clearly, individual domains with a high degree of lateral order can be detected thereby outranging, to the best of our knowledge, all previous reports on transition-metal based micellar arrays deposited after directly loading the precursor salt in solution.

Since inorganic nanostructures arising from salt-loaded micellar monolayers represent our final goal, optimized in situ plasma procedures developed earlier ${ }^{\mathbf{4 , 1 9 , 2 3 , 4 5}}$ were applied to completely remove all organic constituents as evidenced by in situ XPS measurements. Fig. 3 summarizes AFM results (a-d) together with extracted particle size distributions $(\mathrm{e}-\mathrm{h})$ after plasma treatment of the different micellar arrays presented in Fig. 2a-d. Obviously, using the significantly lower molecular weight of hP2VP(1200) carriers (as compared to the P2VP(90 000) blocks forming the micellar cores) not only deteriorates the hexagonal order of the different micellar monolayers (Fig. 2a and b), but also leads to highly disordered arrays of nanoparticles (Fig. 3a and b) accompanied by rather broad particle-size distributions (Fig. $3 e$ and f) thus rendering sizeselection impossible. In clear contrast, size-selection together with hexagonal medium-range order can be achieved for the resulting nanoparticle array if $\mathrm{hP} 2 \mathrm{VP}(97$ 000) molecules are used as carriers to load the corresponding micellar cores. Consequently, arrays of well-separated, size-selected transitionmetal based nanoparticles with unprecedented lateral order are now available representing a crucial pre-requisite to study their size-dependent physical and chemical properties.

It is worth mentioning that the inferior size distribution observed when using hP2VP(1200) molecules cannot be characterized by means of a Gaussian profile. The reason for this is that homopolymers having low molar mass are known to be distributed uniformly in the corresponding domains in a BCPhomopolymer system, with a considerable amount still left in 

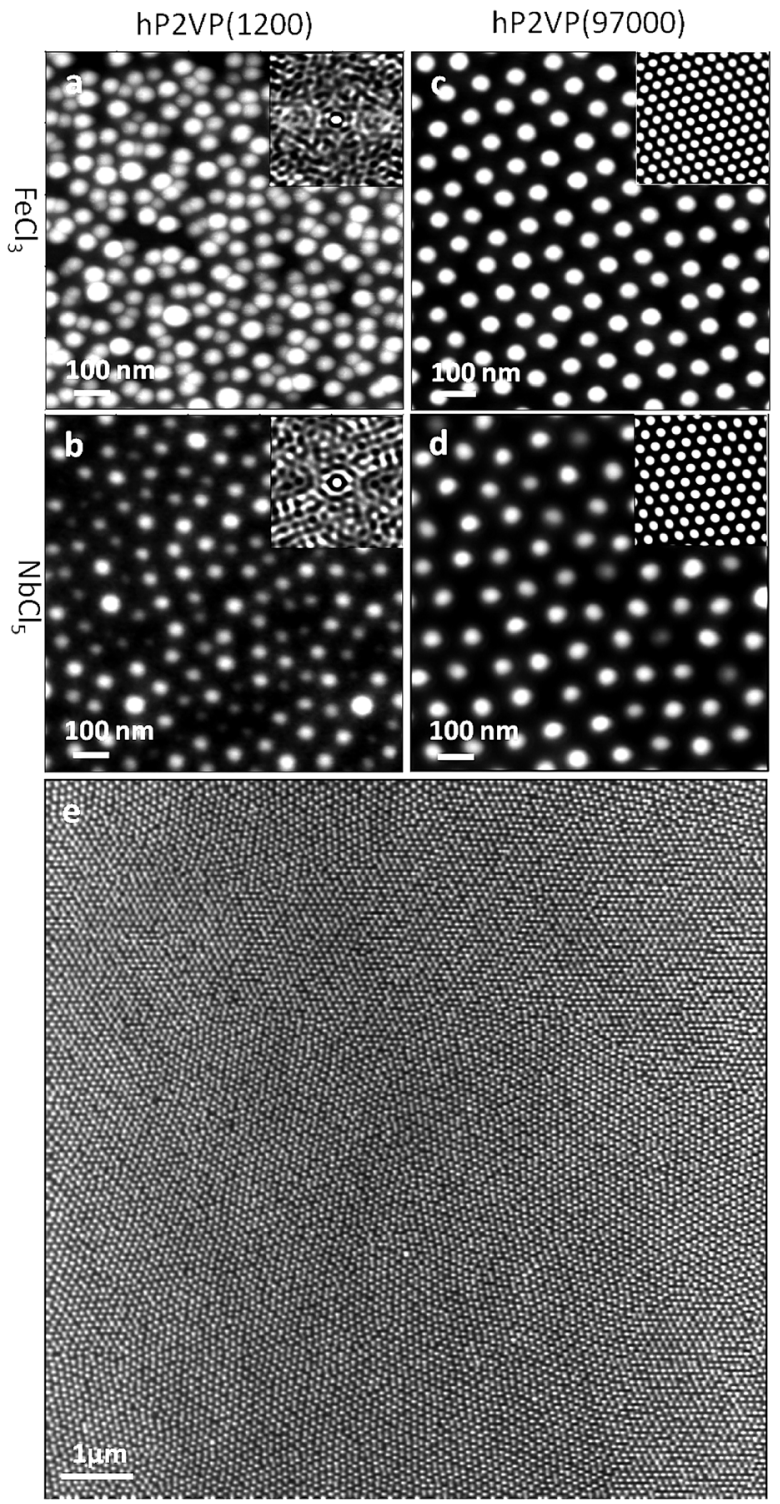

Fig. 2 AFM images and related auto-covariance functions (insets) of micellar patterns obtained from PS(185 000)-b-P2VP(90 000) based solutions that are loaded by $\mathrm{FeCl}_{3}$ and $\mathrm{NbCl}_{5}$, using hP2VP(1200) (a and b) and hP2VP(97 000) (c and d) as precursor carriers; (e) represents a larger scan range of the sample shown in (c).

the continuous phase (the solvent). ${ }^{46}$ Consequently, during the transfer of micelles from solution to a substrate by dip-coating, homopolymer molecules loaded with metal salts will also be deposited within inter-micellar regions resulting in the formation of small clusters after plasma treatment. On the other hand, as hP2VP(97 000) has a molecular weight comparable to the P2VP(90 000) block, it will be confined to the micellar core with a distribution which peaks at the centre of the core ${ }^{\mathbf{4 0}}$ thus avoiding random deposition from the solvent during deposition on a substrate. Hence, for a precise control of the monodispersity of the resulting nanoparticle ensembles, the molecular weight of the hP2VP carrier should not deviate too much from that of the P2VP bock within the micellar nanoreactor. When exploiting our new technique, high quality arrays can be
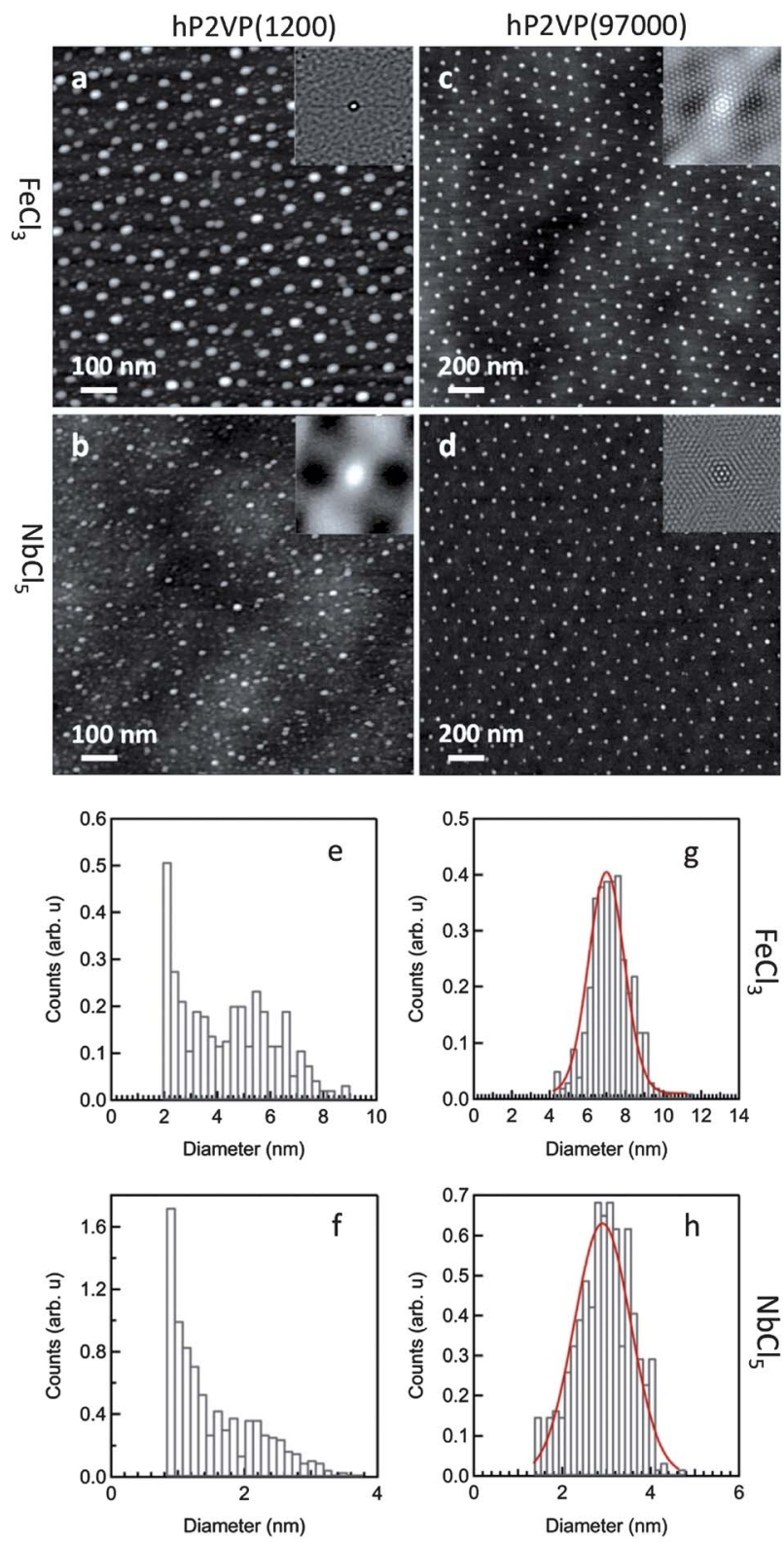

Fig. 3 AFM images $(a-d)$ and size distributions (e-h) of iron oxide (a, c, e and $g$ ) as well as niobium oxide (b, $d, f$ and $h$ ) nanoparticles obtained after oxygen plasma treatment of micellar monolayers prepared using hP2VP(1200) (left row) and hP2VP(97 000) (right row) as carriers for the metal salts. Assuming Gaussian profiles, average particle diameters of $7.0 \pm 0.66 \mathrm{~nm}(\mathrm{~g})$ and $3.0 \pm 0.45 \mathrm{~nm}(\mathrm{~h})$ can be derived for the different material systems.

expected in the future for a variety of transition-metal based nanoparticle systems which now start to compete in array quality with $\mathrm{Au}$ nanoparticle ensembles, ${ }^{\mathbf{1 9 , 2 3 , 4 7}}$ the latter representing the most successful benchmark system which can easily be synthesized even using the conventional micellar approach.

In order to better understand the interaction between the metal precursor and the homopolymer, high-resolution XPS measurements were performed on $\mathrm{FeCl}_{3}$-loaded hP2VP (loading ratio 0.3 ) to study the chemical interplay between the involved species. In this particular case, recent XAFS results unravelling the interaction of $\mathrm{FeCl}_{3}$ with the core of PS- $b$-P2VP micelles 
could serve as a reference: Fe was found to be tetrahedrally coordinated by three $\mathrm{Cl}$ ions (thus implying an oxidation state of $\mathrm{Fe}^{3+}$ ) and, furthermore, to interact with single $\mathrm{N}$ atoms (i.e., single pyridine units). Consequently, an oxidation state of $3+$ could also be anticipated for the iron after loading this salt to the homopolymer. In addition, assuming also that each metal ion interacts with one $\mathrm{N}$ atom only, $30 \%$ of all ring nitrogen atoms should be affected according to the loading ratio of 0.3 .

Such a behaviour can indeed be observed in Fig. 4a which presents the N-1s core-level region of salt-loaded hP2VP. Clearly, two components can be identified at binding energies of 399.5 $\mathrm{eV}$ and $402 \mathrm{eV}$. While the first can be assigned to $\mathrm{N}$ atoms in an organic matrix (C-N type bonds) ${ }^{48}$ thus representing pyridine units not being affected by the metal salt, the latter component is induced by the interaction between ring $\mathrm{N}$ atoms and the positively charged metal ions inducing a shift towards a higher binding energy. By integrating the peak areas of both components, a spectral weight of the higher binding energy line with respect to the total $\mathrm{N}-1 \mathrm{~s}$ intensity of exactly $30 \%$ is obtained, thus evidencing the complexation of single $\mathrm{N}$ atoms by the metal ions as found for PS- $b$-P2VP micelles. Fig. $4 \mathrm{~b}$ provides additional proof for such a behaviour, as an oxidation state of $3+$ can be extracted for the Fe by using the binding energy of the Fe- $2 \mathrm{p}_{3 / 2}$ core line as a probe. Consequently, the chemical interaction between the metal salt and the pyridine units is
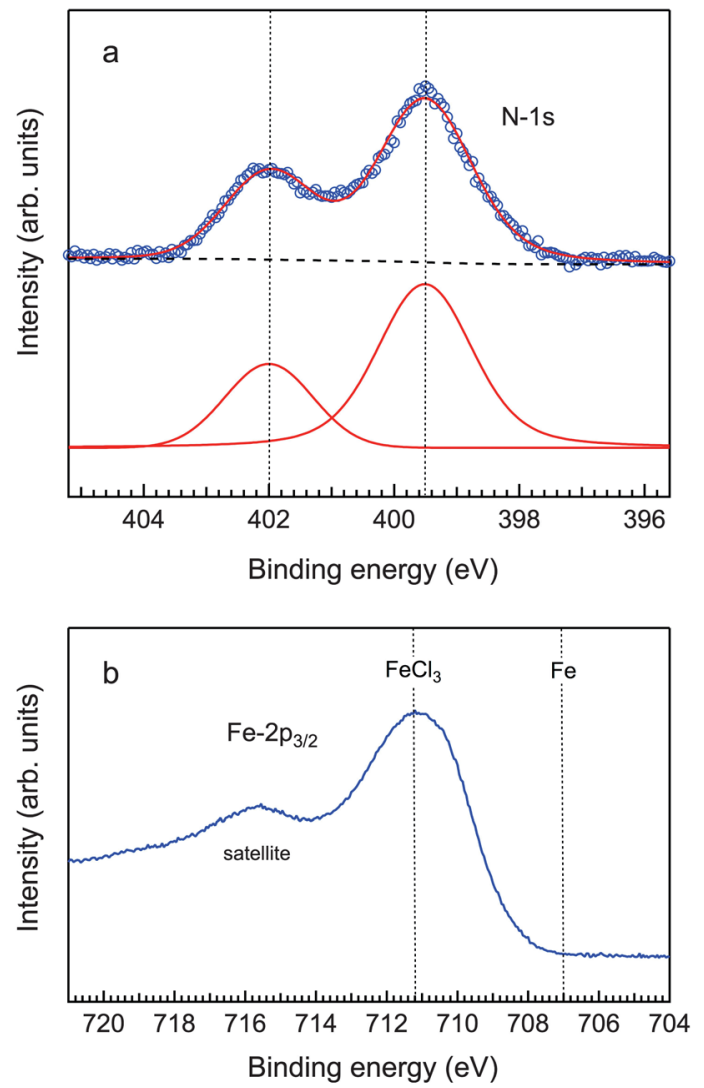

Fig. 4 XPS core level spectra acquired on $\mathrm{FeCl}_{3}$-loaded hP2VP (loading ratio 0.3$)$ : $\mathrm{N}-1$ s binding energy range revealing 2 components (a) and $\mathrm{Fe}-2 \mathrm{p}_{3 / 2}$ core level region giving evidence for the conservation of the oxidation state of the Fe after metal-organic complex formation (b). found to be very similar for both systems (h2VP and PS- $b$-P2VP). This could be one the reasons why these two systems can easily join together to form homogeneously mixed, salt-loaded micellar aggregates in our experiments.

As mentioned earlier, in the case of $\mathrm{Nb}$, a maximum loading ratio of 0.18 was found experimentally, thus limiting the resulting nanoparticle size to $3.0 \mathrm{~nm}$. Interestingly enough, after the micelles are loaded with the precursor-hybridized hP2VP, they can take up additional metal salt without being distorted, which allows the particle size to be further increased while sustaining the hexagonal order. This, in fact, could be anticipated as the P2VP blocks forming a micellar core are also capable of binding polar metal salts. To demonstrate this point, additional $\mathrm{NbCl}_{5}$ (loading ratio 0.5 with respect to the diblock copolymer) was added to the solution which yielded the sample presented in Fig. 3d-h. First results of such an approach are shown in Fig. 5, depicting AFM data (a) as well as the corresponding size distribution (b) measured on the final nanoparticle ensembles. Clearly, this second loading step permits the increase of the particle size from $3.0 \pm 0.43 \mathrm{~nm}$ to $8.1 \pm 1.0 \mathrm{~nm}$, while still maintaining a high degree of hexagonal order. Even though the mechanism behind is not fully clear yet, the presence of salt-hybridized homopolymer chains within the micellar core seems to improve the stability of the micellar compartment, making it easier for subsequently added precursor salt to be homogeneously distributed among (equally-sized) micellar nanoreactors. Such a stabilizing effect can also be exploited to synthesize ordered arrays of transition metal-based alloy nanoparticles by using a different type of metal precursor during the second loading step (to be published elsewhere).
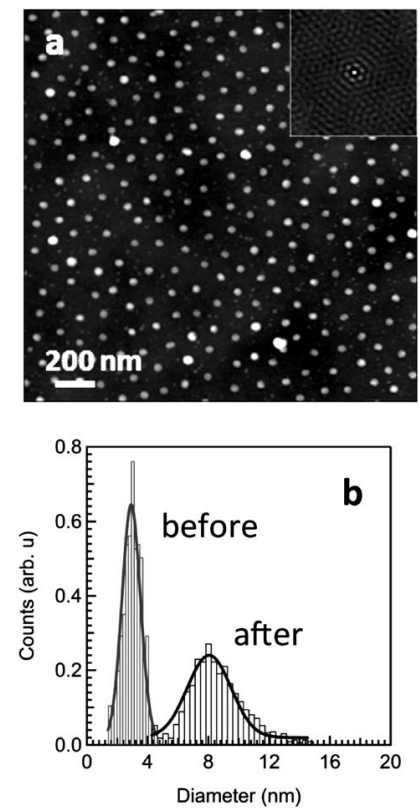

Fig. 5 AFM image (a) and related size distribution (b) of nanoparticles produced by adding additional $\mathrm{NbCl}_{5}$ to a micellar solution which has been pre-loaded by salt-decorated hP2VP (loading ratio 0.18 ). Clearly, the size of the resulting particles is increased without a significant loss of hexagonal order. 
Finally, the question of what makes the difference between shorter or longer H2VP chains exploited as nanocarriers for the metal precursor needs to be addressed. Intuitively, a better performance of shorter hP2VP nanocarriers could be anticipated due to an enhanced diffusion towards the micellar cores while passing the network of PS blocks forming the shell. A better solubility of shorter homopolymer chains within diblock copolymer micelles was indeed found experimentally ${ }^{\mathbf{4 9 , 5 0}}$ and verified theoretically $^{38}$ in clear contrast to our experimental findings. Hence, it might be speculated that the pre-loading of homopolymer chains with metal salts (thereby strongly increasing their polar character) might be responsible for the improved storage of longer chains within the micellar nanoreactors observed here. However, only a full theoretical treatment of this problem will allow the underlying effects to be unraveled.

Based on the approach described so far, nanoparticle arrays with excellent hexagonal order have been achieved for $\mathrm{Fe}, \mathrm{Nb}$, as well as Mo ranging in size between about 1 and $10 \mathrm{~nm}$. The size can be controlled by the precise amount of precursor salt added to the homopolymer solution and, eventually (as in the case of $\mathrm{Nb}$ ), added to the homopolymer-stabilized micellar solution within a second loading step. This size range is comparable with what can be achieved by direct loading of the metal salt into the micellar cores. ${ }^{1923}$ A nice benchmark system for the direct loading approach is Au, which can also be deposited successfully into highly ordered nanoparticle arrays using our new method. Thus, the homopolymer technique seems to be applicable to a broader range of materials (transition metals and noble metals) making it an attractive alternative to the currently existing techniques of direct loading of micellar nanoreactors. It is worth mentioning that, in the latter case, an exciting new method has been reported recently ${ }^{51}$ allowing deposition of nanoparticle arrays on an unprecedented short time scale.

\section{Conclusion}

In conclusion, we have demonstrated a new and facile approach to prepare well-ordered arrays of transition metal-based inorganic nanoparticles at surfaces. Using hP2VP of appropriate length as molecular carriers to transport precursor salts into PS- $b$-P2VP micelles significantly improves the degree of lateral order within the final nanoparticle arrays. Thus, a significantly extended range of well-organized, well-separated, size-selected metal and metal oxide nanostructures become available in the future. This opens exciting new opportunities to study the fundamental properties of nanostructures at the transition from clusters to solids, where well-defined particle sizes and inter-particle spacings are of crucial importance like in optics, catalysis, and magnetism.

\section{Acknowledgements}

Financial support by the Flemish Research Foundation (FWO) within the Odysseus program, the FWO projects G.0456.12, G.0346.09N, and the Methusalem project "NANO" is gratefully acknowledged. We furthermore thank J. Baccus, T. Jacobs and J. Artois for technical support and A. Ethirajan for fruitful discussions.

\section{References}

1 B. R. Cuenya, S.-H. Baeck, T. F. Jaramillo and E. W. McFarland, J. Am. Chem. Soc., 2003, 125, 12928-12934.

2 L. Ono and B. Roldán-Cuenya, Catal. Lett., 2007, 113, 86-94.

3 S. Mostafa, F. Behafarid, J. R. Croy, L. K. Ono, L. Li, J. C. Yang, A. I. Frenkel and B. R. Cuenya, J. Am. Chem. Soc., 2010, 132, 15714-15719.

4 H.-G. Boyen, G. Kästle, F. Weigl, B. Koslowski, C. Dietrich, P. Ziemann, J. P. Spatz, S. Riethmuller, C. Hartmann, M. Möller, G. Schmid, M. G. Garnier and P. Oelhafen, Science, 2002, 297, 1533-1536.

5 H.-G. Boyen, A. Ethirajan, G. Kästle, F. Weigl, P. Ziemann, G. Schmid, M. G. Garnier, M. Buttner and P. Oelhafen, Phys. Rev. Lett., 2005, 94, 016804.

6 A. Ethirajan, U. Wiedwald, H.-G. Boyen, B. Kern, L. Y. Han, A. Klimmer, F. Weigl, G. Kästle, P. Ziemann, K. Fauth, J. Cai, R. J. Behm, A. Romanyuk, P. Oelhafen, P. Walther, J. Biskupek and U. Kaiser, Adv. Mater., 2007, 19, 406-410.

7 M. Pelton, J. Aizpurua and G. Bryant, Laser Photonics Rev., 2008, 2, 136-159.

8 J. N. Anker, W. P. Hall, O. Lyandres, N. C. Shah, J. Zhao and R. P. V. Duyne, Nat. Mater., 2008, 7, 442-453.

9 C. Hinderling, Y. Keles, T. Stöckli, H. F. Knapp, T. de los Arcos, P. Oelhafen, I. Korczagin, M. A. Hempenius, G. J. Vancso, R. Pugin and H. Heinzelmann, Adv. Mater., 2004, 16, 876-879.

10 M. Haupt, A. Ladenburger, R. Sauer, K. Thonke, R. Glass, W. Roos, J. P. Spatz, H. Rauscher, S. Riethmüller and M. Möller, J. Appl. Phys., 2003, 93, 6252-6257.

11 B. N. Mbenkum, A. S. Schneider, G. Schütz, C. Xu, G. Richter, P. A. van Aken, G. Majer and J. P. Spatz, ACS Nano, 2010, 4, 1805.

12 B. Geiger, J. P. Spatz and A. D. Bershadsky, Nat. Rev. Mol. Cell Biol., 2009, 10, 21-33.

13 J. Huang, S. V. Gräter, F. Corbellini, S. Rinck, E. Bock, R. Kemkemer, H. Kessler, J. Ding and J. P. Spatz, Nano Lett., 2009, 9, 1111-1116.

14 A. C. Pearson, E. Pound, A. T. Woolley, M. R. Linford, J. N. Harb and R. C. Davis, Nano Lett., 2011, 11, 1981-1987.

15 A. M. Popa, B. Wenger, E. Scolan, G. Voirin, H. Heinzelmann and R. Pugin, Appl. Surf. Sci., 2009, 256, S12-S17.

16 J. P. Spatz, A. Roescher and M. Möller, Adv. Mater., 1996, 8, 337-340.

17 M. Park, C. Harrison, P. M. Chaikin, R. A. Register and D. H. Adamson, Science, 1997, 276, 1401-1404.

18 S. Förster and M. Antonietti, Adv. Mater., 1998, 10, 195-217. 19 J. P. Spatz, S. Mössmer, C. Hartmann, M. Möller, T. Herzog, M. Krieger, H.-G. Boyen, P. Ziemann and B. Kabius, Langmuir, 2000, 16, 407-415.

20 T. Thurn-Albrecht, J. Schotter, C. A. Kastle, N. Emley, T. Shibauchi, L. Krusin-Elbaum, K. Guarini, C. T. Black, M. T. Tuominen and T. P. Russell, Science, 2000, 290, 2126-2129.

21 J. Y. Cheng, A. M. Mayes and C. A. Ross, Nat. Mater., 2004, 3, 823-828. 
22 S. Park, D. H. Lee, J. Xu, B. Kim, S. W. Hong, U. Jeong, T. Xu and T. P. Russell, Science, 2009, 323, 1030-1033.

23 G. Kästle, H.-G. Boyen, F. Weigl, G. Lengl, T. Herzog, P. Ziemann, S. Riethmuller, O. Mayer, C. Hartmann, J. P. Spatz, M. Möller, M. Ozawa, F. Banhart, M. G. Garnier and P. Oelhafen, Adv. Funct. Mater., 2003, 13, 853-861.

24 J. Bansmann, S. Kielbassa, H. Hoster, F. Weigl, H.-G. Boyen, U. Wiedwald, P. Ziemann and R. J. Behm, Langmuir, 2007, 23, 10150-10155.

25 R. Abe, K. Shinohara, A. Tanaka, M. Hara, J. N. Kondo and K. Domen, Chem. Mater., 1997, 9, 2179-2184.

26 H. Kominami, K. Oki, M. Kohno, S. Onoue, Y. Kera and B. Ohtani, J. Mater. Chem., 2001, 11, 604-609.

27 K. Tanabe, Catal. Today, 2003, 78, 65-77.

28 J. S. Jang, H. G. Kim, V. R. Reddy, S. W. Bae, S. M. Ji and J. S. Lee, J. Catal., 2005, 231, 213-222.

29 P. Charvin, S. Abanades, G. Flamant and F. Lemort, Energy, 2007, 32, 1124-1133.

30 M. A. Aegerter, M. Schmitt and Y. Guo, Int. J. Photoenergy, 2002, 4, 1-10.

31 C.-S. Lee, D. Kwon, J. E. Yoo, B. G. Lee, J. Choi and B. H. Chung, Sensors, 2010, 10, 5160-5170.

32 W. X. Zhang, J. Nanopart. Res., 2003, 5, 323-332.

33 J. Zhou, H. Song, X. Chen, L. Zhi, S. Yang, J. Huo and W. Yang, Chem. Mater., 2009, 21, 2935-2940.

34 J. P. Spatz, T. Herzog, S. Mössmer, P. Ziemann and M. Möller, Adv. Mater., 1999, 11, 149-153.

35 N. H. Agnew, J. Polym. Sci., Polym. Chem. Ed., 1976, 14, 28192830.

36 D. H. Lee, H. Y. Kim, J. K. Kim, J. Huh and D. Y. Ryu, Macromolecules, 2006, 39, 2027-2030.

37 D. H. Lee, S. H. Han, W. Joo, J. K. Kim and J. Huh, Macromolecules, 2008, 41, 2577-2583.
38 D. Izzo and C. M. Marques, J. Phys. Chem. B, 2005, 109, 61406145.

39 T. Hashimoto, H. Tanaka and H. Hasegawa, Macromolecules, 1990, 23, 4378-4386.

40 A. Mayes, T. Russell, S. Satija and C. Majkrzak, Macromolecules, 1992, 25, 6523-6531.

41 Y. Matsushita, N. Torikai, Y. Mogi, I. Noda and C. Han, Macromolecules, 1993, 26, 6346-6349.

42 B. Loewenhaupt, A. Steurer, G. P. Hellmann and Y. Gallot, Macromolecules, 1994, 27, 908-916.

43 U. Jeong, D. Y. Ryu, D. H. Kho, D. H. Lee, J. K. Kim and T. P. Russell, Macromolecules, 2003, 36, 36263634 .

44 K. O. Stuen, C. S. Thomas, G. Liu, N. Ferrier and P. F. Nealey, Macromolecules, 2009, 42, 5139-5145.

45 H.-G. Boyen, G. Kästle, K. Zurn, T. Herzog, F. Weigl, P. Ziemann, O. Mayer, C. Jerome, M. Möller, J. P. Spatz, M. G. Garnier and P. Oelhafen, Adv. Funct. Mater., 2003, 13, 359-364.

46 M. P. Pépin and M. D. Whitmore, Macromolecules, 2000, 33, 8654-8662.

47 R. E. Palmer, S. Pratontep and H.-G. Boyen, Nat. Mater., 2003, 2, 443-448.

48 J. F. Moulder, W. F. Stickle, P. E. Sobol and K. D. Bomben, Handbook of X-ray Photoelectron Spectroscopy, Physical Electronics, Inc., Eden Prairie, MN 55344, 1992.

49 J. R. Quintana, R. A. Salazar and I. Katime, Macromolecules, 1994, 27, 665-668.

50 J. R. Quintana, M. D. Janez and I. Katime, Polym. Int., 1996, 40, 123-128.

51 A. Ethirajan, S. Punniyakoti, M. D'Olieslaeger, P. Wagner and H.-G. Boyen, Angew. Chem., Int. Ed., 2013, 52, 97099713. 